\title{
Locking a molecular bond: A case study of CsI
}

Szakács, Tamás; Amstrup, Bjarne; Gross, Peter; Kosloff, Ronnie; Rabitz, Herschel; Lörincz, András

Published in:

Physical Review A

Link to article, DOI:

10.1103/PhysRevA.50.2540

Publication date:

1994

Document Version

Publisher's PDF, also known as Version of record

Link back to DTU Orbit

Citation (APA):

Szakács, T., Amstrup, B., Gross, P., Kosloff, R., Rabitz, H., \& Lörincz, A. (1994). Locking a molecular bond: A case study of CsI. Physical Review A, 50(3), 2540-2547. https://doi.org/10.1103/PhysRevA.50.2540

\section{General rights}

Copyright and moral rights for the publications made accessible in the public portal are retained by the authors and/or other copyright owners and it is a condition of accessing publications that users recognise and abide by the legal requirements associated with these rights.

- Users may download and print one copy of any publication from the public portal for the purpose of private study or research.

- You may not further distribute the material or use it for any profit-making activity or commercial gain

- You may freely distribute the URL identifying the publication in the public portal 


\title{
Locking a molecular bond: A case study of CsI
}

\author{
Tamás Szakács, ${ }^{1}$ Bjarne Amstrup, ${ }^{2, *}$ Peter Gross,${ }^{3}$ Ronnie Kosloff, ${ }^{4}$ Herschel Rabitz, ${ }^{3}$ and András Lőrincz ${ }^{1, \dagger}$ \\ ${ }^{1}$ Department of Photophysics, Institute of Isotopes of the Hungarian Academy of Sciences, P.O. Box 77, \\ H-1525 Budapest, Hungary \\ ${ }^{2}$ Chemistry Department B, Technical University of Denmark, DTU-301, DK-2800 Lyngby, Denmark \\ ${ }^{3}$ Department of Chemistry, Princeton University, Princeton, New Jersey 08544 \\ ${ }^{4}$ Department of Physical Chemistry and the Fritz Haber Research Center, The Hebrew University,
} Jerusalem 91904, Israel

(Received 22 March 1994)

\begin{abstract}
This paper treats the problem of locking a molecular bond at a length other than the equilibrium distance, with the help of optical electric fields. Locking conditions for single-color fields are examined, and slowly decaying locked wave functions are sought. These wave functions are then used as target functions in an optimal control procedure. The resultant solution is an optimal field that creates a wave function as close as possible to the target function, followed by the application of a locking single-color field that can keep a large part of this wave function at the given position. An objective of the approach is to eventually extend the optimal control theory to the preparation of molecules in quasistationary nonequilibrium states for further study by spectroscopic or other means. Results are reported for a model of CsI with two potential-energy surfaces, one with a repulsive character.
\end{abstract}

PACS number(s): 42.50.Hz, 33.80.-b

\section{INTRODUCTION}

Optimal control of molecular processes with shaped ultrashort laser pulses is the focus of much recent research interest [1-44]. In these examples the quantum system is initially in a given state or statistical mixture of states and the timing of the dynamics is given by the laser pulse. In other words, the system to be controlled is waiting for the controller. The problems studied are typically the creation of a specific final state or enhancing the yield of a photochemical reaction.

The perspective taken in the present paper is distinct from that of the previous work. Here we intend to demonstrate the possibility of locking a molecular bond at a length other than its natural equilibrium point. The ability to achieve such a goal in the laboratory could have a number of applications. In particular, one could consider performing vibronic spectroscopy on such a locked molecule, and the resultant Franck-Condon transitions could access interesting states, from the newly locked initial state. Second, potentially a number of interesting scattering processes might also become available for study. This latter category of problems inherently involves events whose timing is statistically distributed. The ability to lock bonds opens up the dynamical study of unusually prepared molecular configurations. The goal

${ }^{*}$ Electronic address: kebjarne@vm.uni-c.dk Address after October 1, 1994: Chemistry Department B, Technical University of Denmark, DTU-206, DK-2800 Lyngby, Denmark.

${ }^{\dagger}$ Electronic address: lorincz@iserv.iki.kfki.hu of this paper is to make one step in this direction by considering bond length locking in diatomic molecules. The problem of influencing scattering cross sections is complex since (i) it involves at least three-body interactions, (ii) known Hamiltonians may be crude approximations, (iii) locking may break down when scattering starts since the effect of the field may be very different during scattering, (iv) scattering may involve an ensemble of molecules with Doppler broadening and an energy distribution, instead of a well defined quantum system, etc. Theoretical studies of scattering processes with locked molecules thus can only provide approximations even with stateof-the-art methods. However, laboratory optimization, first suggested by Judson and Rabitz [23] and a subject of recent interest $[32,33,44]$ for optimal control of chemical processes, may prove to be viable for optimal control of scattering processes too. Theoretical work can provide the initial designs to gain insight for eventual experimental optimization. This is a goal of the present study.

\section{GENERAL CONSIDERATIONS}

The optimal control approach to design shaped laser pulses is now firmly established; details can be found in $[6,9,11]$; here only a brief description will be given.

The equation of motion in our system is the timedependent Schrödinger equation $\left(\partial_{t} \equiv \frac{\partial}{\partial t}\right)$

$$
i \hbar \partial_{t}|\Psi(t)\rangle \equiv i \hbar|\dot{\Psi}(t)\rangle=\widehat{H}|\Psi(t)\rangle
$$

Within the semiclassical radiation and adiabatic dynam- 
ical approximations, the Hamiltonian may be written as

$$
\widehat{H}=\widehat{H}(\vec{x}, t)=\left(\begin{array}{cc}
\widehat{H}_{u}(\vec{x}) & -\mu(\vec{x}) \mathcal{E}(t) \\
-\mu(\vec{x}) \mathcal{E}(t) & \widehat{H}_{g}(\vec{x})
\end{array}\right)
$$

and

$$
|\Psi(t)\rangle=\int d x|x\rangle \Psi(x, t)=\left(\begin{array}{c}
\left|\psi_{u}\right\rangle \\
\left|\psi_{g}\right\rangle
\end{array}\right)
$$

The subscript $g$ refers to the "ground" or initial state potential energy surface and $u$ correspondingly refers to the "upper" or interacting state potential-energy surface. $\widehat{H}_{i}=\widehat{T}+V_{i}(\vec{x})$, where $\widehat{T}=\hat{p}^{2} / 2 m$ is the kinetic-energy operator and $V_{i}$ is the corresponding potential energy. $\mu$ is the projection of the transition dipole moment operator along the direction of the electric field polarization and $\mathcal{E}(t)$ represents the amplitude of the classical electric field.

The objective to be optimized for this problem may be given as (see, for example, $[6,9,13,18,31]$ )

$$
\begin{aligned}
\mathcal{J}= & \left\langle\Psi\left(t_{f}\right)|\widehat{P}| \Psi\left(t_{f}\right)\right\rangle-\lambda_{e} \int_{t_{0}}^{t_{f}} \mathcal{E}^{2}(t) d t \\
& +\frac{i}{\hbar} \int_{t_{0}}^{t_{f}}\left(\left\langle\Phi\left|i \hbar \partial_{t}-\widehat{H}\right| \Psi\right\rangle-\text { c.c. }\right) d t .
\end{aligned}
$$

The first term describes a final wave function $\Psi\left(t_{f}\right)$, projected with the help of an operator $\widehat{P}$ onto the targeted state. The projection operator may be written in the following form without loss of generality:

$$
\widehat{P}=\left(\begin{array}{cc}
a_{u}\left|\chi_{u}\right\rangle\left\langle\chi_{u}\right| & 0 \\
0 & a_{g}\left|\chi_{g}\right\rangle\left\langle\chi_{g}\right|
\end{array}\right)
$$

with $a_{i} \geq 0, i=u, g$, and

$$
a_{g}+a_{u}=1 \text {. }
$$

The second term in Eq. (2.4) is a penalty for the integrated energy of the electric field. It limits the electric energy used in the optimization procedure in balance with the targeted wave function. That balance may be tuned with the help of $\lambda_{e}$, with higher $\lambda_{e}$ values corresponding to a more "strict policy" on the electric field energy. The third term represents the constraint that the system should always satisfy the equation of motion (2.1). This term contains the generalized Lagrange multiplier $\Phi(x, t)$. In analogy with $\Psi, \Phi$ is a two-component function assuming the form

$$
|\Phi\rangle=\left(\begin{array}{l}
\left|\phi_{u}\right\rangle \\
\left|\phi_{g}\right\rangle
\end{array}\right)
$$

Application of the calculus of variation leads to (see, e.g., $[6,9,13,18]$ ) a set of control equations: the Schrödinger equation for the wave function as well as the generalized Lagrange multiplier, including an initial condition for the wave function and a final condition for the generalized Lagrange multiplier:

$$
\begin{gathered}
i \hbar \partial_{t}|\Psi(t)\rangle=\widehat{H}|\Psi(t)\rangle, \\
i \hbar \partial_{t}|\Phi(t)\rangle=\widehat{H}|\Phi(t)\rangle, \\
\left|\Psi\left(t_{0}\right)\right\rangle=\left(\begin{array}{c}
0 \\
\left|\psi_{g}\left(t_{0}\right)\right\rangle
\end{array}\right), \\
\left|\Phi\left(t_{f}\right)\right\rangle=\widehat{P}\left|\Psi\left(t_{f}\right)\right\rangle .
\end{gathered}
$$

Equations (2.8)-(2.11) are further linked by the equation for the optimal electric field $\mathcal{E}$,

$$
\mathcal{E}(t)=-\frac{1}{\lambda_{e} \hbar} \operatorname{Im}\left(\left\langle\phi_{g}|\mu| \psi_{u}\right\rangle-\left\langle\psi_{g}|\mu| \phi_{u}\right\rangle\right)
$$

The Krotov iterative scheme can be used for solving these coupled equations for $\mathcal{E}(t)[39,26,9]$. The iteration procedure has the following steps.

(1) Make an initial guess for the electric field.

(2) Integrate the Schrödinger equation for $\Psi$ forward up to time $t_{f}$ starting from the initial state of the system.

(3) Apply the projection operator $\widehat{P}$ to the final time wave function according to Eq. (2.11) to obtain $\Phi\left(t_{f}\right)$.

(4) Propagate $\Phi$ backward in time to obtain $\Phi\left(t_{0}\right)$.

(5) Calculate $\mathcal{E}\left(t_{0}\right)$ according to Eq. (2.12) from $\Phi\left(t_{0}\right)$ and $\Psi\left(t_{0}\right)$. Then calculate $\Phi\left(t_{0}+d t\right)$ using the old electric field generated in the previous iteration step and calculate $\Psi\left(t_{0}+d t\right)$ using the newly generated electric field. In this manner $\Psi$ and $\Phi$ are propagated forward up to the final time calculating the new electric field at each time step.

(6) Go back to (3) and repeat (3)-(5) until the specified convergence criterion is reached.

This procedure was used for the initial iterations. The iteration procedure was found fast compared to the traditional gradient algorithms, but in some cases it became unstable for our problem. In addition, the Krotov method started to develop unreasonably large electric fields at some stage of the procedure for early times of control.

The electric field was monitored and when the breakdown of the Krotov iterative scheme started to happen it was replaced by a gradient method for further iterations. In this way the Krotov method was used for finding an initial guess for a local gradient search. For gradient methods it is easy to implement a time-dependent penalty function (see, e.g., $[6,31]$ ). In this case the objective may be written as

$$
\begin{aligned}
\mathcal{J}_{w}= & \left\langle\Psi\left(t_{f}\right)|\widehat{P}| \Psi\left(t_{f}\right)\right\rangle-\lambda_{e} \int_{t_{0}}^{t_{f}} W(t) \mathcal{E}^{2}(t) d t \\
& +\frac{i}{\hbar} \int_{t_{0}}^{t_{f}}\left(\left\langle\Phi\left|i \hbar \partial_{t}-\widehat{H}\right| \Psi\right\rangle-\text { c.c. }\right) d t .
\end{aligned}
$$

The weight function $W(t)$ was chosen high and quickly decreasing at the edges of the time region and 1 in be- 
tween. This form of the objective leads to the following gradient:

$$
\begin{aligned}
\frac{\delta \mathcal{J}_{w}}{\delta(\mathcal{E}(t))}= & -2 \lambda_{e} W(t) \mathcal{E}(t) \\
& -\frac{2}{\hbar} \operatorname{Im}\left(\left\langle\phi_{g}|\mu| \psi_{u}\right\rangle-\left\langle\psi_{g}|\mu| \phi_{u}\right\rangle\right)
\end{aligned}
$$

This gradient was then used in the Fletcher-Reeves version of the conjugate gradient method [45] combined with a line search as described in [31].

The ground and upper parts of the target wave function $\chi_{u}$ and $\chi_{g}$ of the projection operator were found through the locking procedure to be described in the next section.

\section{WAVE-FUNCTION LOCKING}

All calculations were carried out using atomic units: $1 E_{h}=219474.64 \mathrm{~cm}^{-1}, 1 \hbar / E_{h}=0.0241888 \mathrm{fs}, 1 E_{h} /\left(e a_{0}\right)$ $=5.14221 \times 10^{11} \mathrm{~V} / \mathrm{m}$.

A simple model system was studied to get insight into wave-function locking. The model consisted of two linear Born-Oppenheimer (BO) curves of opposite slopes. In treating the optical coupling between the $\mathrm{BO}$ surfaces we assumed that the rotating-wave approximation is valid. Notationally, we distinguish between the model of two linear BO curves and the CsI BO curves by using the subscripts 1 (for positive slope) and 2 (for negative slope) for the linear BO curves and $g$ (ground) and $u$ (upper) for the CsI BO curves.

The time-dependent Schrödinger equation was solved numerically by a split operator method $[46,47]$ or a Lanczos method [46] that uses a spatial grid and fast Fourier transformation (FFT) for computing the Laplacian operator on the grid. In order to allow the evaluation of the Laplacian operator with the help of FFT methods (i.e., under periodic boundary conditions) parts of the wave function that could escape from the "region of interest"

$$
V^{d}=\left(\begin{array}{cc}
\sqrt{(\mu \mathcal{E})^{2}+\left[\delta\left(x-x_{0}\right)\right]^{2}} & 0 \\
0 & -\sqrt{(\mu \mathcal{E})^{2}+\left[\delta\left(x-x_{0}\right)\right]^{2}}
\end{array}\right)
$$

where $\pm \delta$ is the slope of the linear potentials and $x_{0}$ is the crossing point of the surfaces. The diagonalized potentials are shown in Fig. 2. The upper potential well has localized eigenfunctions. One can show that the beats on the wave functions correspond to the frequency differences between the ground state and the excited states of the well. The initial state was made symmetric, i.e., it cannot develop antisymmetric components as a result of propagation and the oscillation frequency was found to correspond to the beat frequency between the ground state and the second excited state. In another run the "cleaned" wave function was slightly displaced, thus allowing us to have an antisymmetric component. The propagation is shown in Fig. 3. This propagation shows oscillations of about half the frequency of Fig. 1.

The beat frequencies and frequency differences be- were "absorbed" by adding an "optical" (i.e., imaginary) tials. It may be worth noting that the optical potential and the electric fields are not related.

The rotating-wave approach in effect shifts the $\mathrm{BO}$ surfaces by the amount corresponding to the applied frefrequency from the exciting field and leaves only the envelope behind. If the envelope is smooth then efficient mixing of the $\mathrm{BO}$ surfaces is possible in the neighborhood of the crossing point of the shifted potentials. To start the computation, the initial wave function on each surface was made of identical Gaussian wave functions faces. The wave functions oscillate heavily as a result of the strong coupling between the surfaces and the acceleration on each surface. Parts of the wave function are leaking out quickly from the potential well created by the two potentials and the interaction between them, but there are parts that have a much longer lifetime. To study the effect, the following procedure was introduced: (a) the wave function was propagated and (b) it was normalized to 1 after each time step. This method was used since the wave function decays and normalization at each time step can avoid this obstacle. Through this method, short lifetime components of the wave function decay while long lifetime components survive. A true propagation after this "cleaning procedure" are shown in Fig. 1. In all cases of linear BO surfaces the wave function of one of the potential surfaces is shown since the other part of the wave function behaves in a similar fashion, but opposite in phase.

The wave functions are oscillating and that is the result of the interference between parts of the wave function. To proceed we note that the Hamiltonian without the kinetic terms may be diagonalized. The kinetic term couples these potentials and they may be considered uncoupled asymptotically in the high field limit. The diagonalized potential is

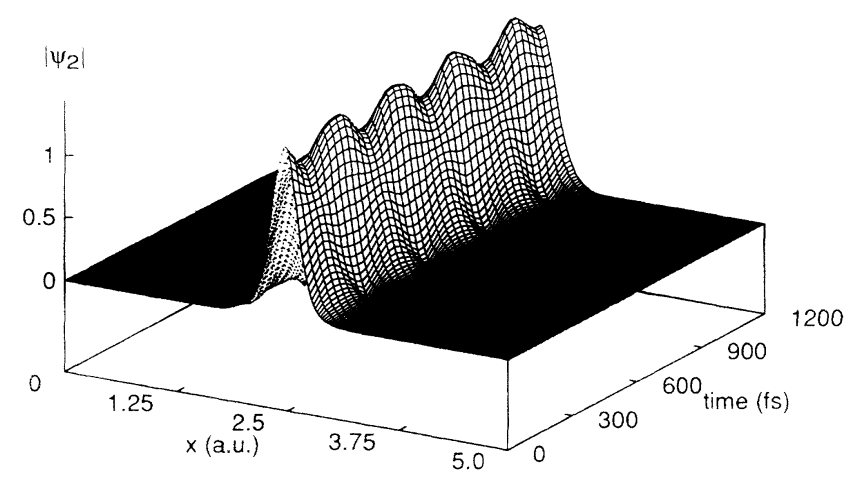

FIG. 1. Evolution of the "cleaned" wave function on BO surface 2. Evolution on BO surface 1 is similar, but opposite in phase. potential [48] to the low energy parts of both linear potenquency of light. At the same time it removes the carrier and the centers placed to the crossing point of the sur- 


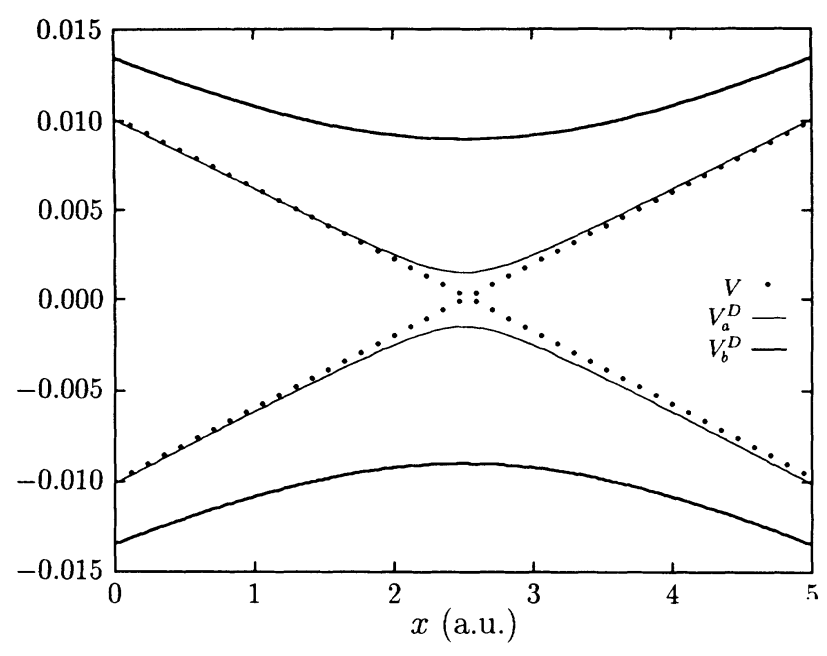

FIG. 2. Diagonalized interacting linear potentials. The slopes of the linear surfaces are $\pm 0.004 E_{h} / a_{0}$. The interacting electric fields are $\mathcal{E}=0.005 \mathrm{a}$.u. for $V_{a}^{D}$ and $\mathcal{E}=0.03 \mathrm{a} . \mathrm{u}$. for $V_{b}^{D}$, respectively.

tween the ground and the second excited states for different slopes and electric field amplitudes are shown in Table I. The beat frequencies were computed by Fourier transforming a time-dependent slice of the wave-function amplitude at a given position. The power spectrum of the Fourier transform of a slice (not shown) exhibits a high peak corresponding to the Rabi flopping frequency. A secondary peak is found corresponding to the beat frequency between the ground state and the second excited state. Table I shows good agreement between the frequency $\omega_{13}$ determined from the bound state eigenvalues and $\omega_{b}$ determined from the Fourier frequency differences above. From the correlation between beat frequencies and the energy differences of the eigenfunctions of the upper part of the diagonalized potentials, the suggested way to find the locked state is to start with the ground state of the upper diagonalized potential and compute the corresponding state for the original potentials, i.e., use the inverse of the diagonalization transformation. The transformed wave function is shown in Fig. 4. The propagation of this wave function on the linear BO surface 2 is given

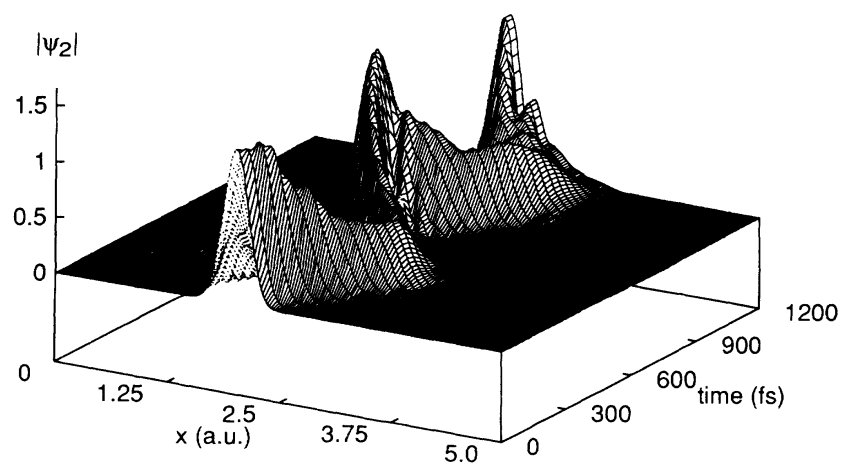

FIG. 3. Evolution of displaced wave function on BO surface
TABLE I. Beat frequencies $\left(\omega_{b}\right)$, frequency difference $\left(\omega_{13}\right)$ for different slopes $(\delta)$, and electric field amplitudes $\left(\mathcal{E}_{0}\right)$. All values are in atomic units except for the corresponding oscillation times (in parentheses), which are in femtoseconds.

\begin{tabular}{cccc}
\hline \hline$\delta$ & $\mathcal{E}_{0}$ & $\omega_{b}$ & $\omega_{13}$ \\
\hline 0.004 & 0.005 & $5.63 \times 10^{-4}(270 \mathrm{fs})$ & $5.47 \times 10^{-4}(278 \mathrm{fs})$ \\
0.004 & 0.03 & $2.53 \times 10^{-4}(600 \mathrm{fs})$ & $2.43 \times 10^{-4}(625 \mathrm{fs})$ \\
0.020 & 0.03 & $11.4 \times 10^{-4}(133 \mathrm{fs})$ & $11.8 \times 10^{-4}(128 \mathrm{fs})$ \\
\hline \hline
\end{tabular}

in Fig. 5. The remaining oscillations are a consequence of the approximation used (e.g., neglecting the kinetic terms in the diagonalization procedure).

In another set of runs it was studied how long one can keep the wave function locked or, in other words, what is the leaking rate at constant field amplitude. Runs were made for different slopes, different electric field strengths, and with true Schrödinger propagation, i.e., without the normalization in each step. Then the region without the optical potential was evaluated and the probability of finding the wave function within this region was computed. In the time interval studied, the decay was fitted by an exponential, the rate of which gives an upper bound on the inverse of the longest lifetime, since the longest lifetime state was not always reached in the computations. The decay rates are shown in Fig. 6. The parameters of the curves are the slopes. Decay rate increases with increasing slope of the potentials and decreases with increasing amplitude of the electric field: The higher the slope of the potentials the more rapid the leakage from the locked region, and the stronger the locking field the more efficiently it blocks the leaking. As may be seen from the figure, reasonable electric fields result in efficient locking. (The value $-2 \times 10^{-5}$ of the figure corresponds approximately to a 1 ps lifetime.) In this work only BO "surfaces" of identical - but opposite in sign - slope were investigated. The problem is, however, very much the same for the case of different slopes. Locking becomes unstable if both BO surfaces are tilted to the same direction or, in other words, if the slopes have identical sign.

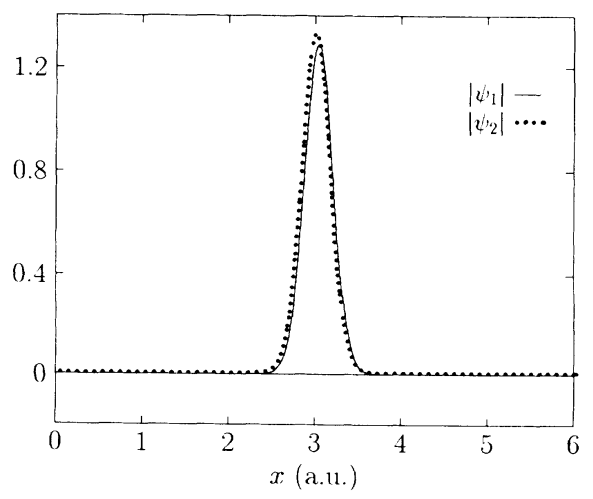

FIG. 4. Target wave functions. (At every point the wave functions are of the same phase.) 


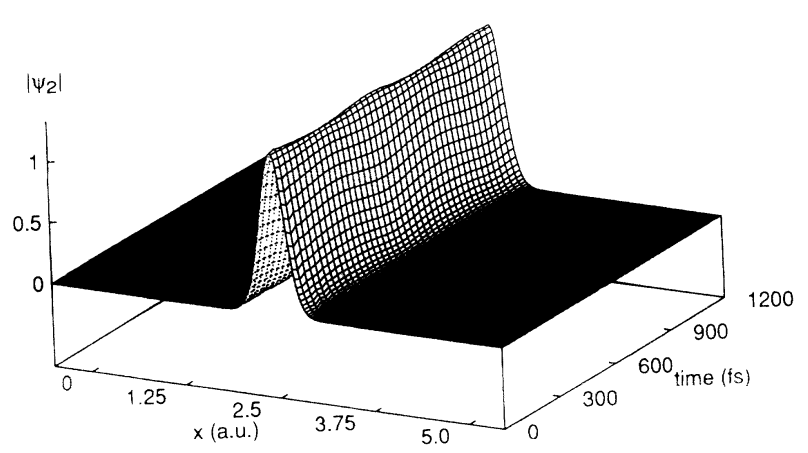

FIG. 5. Evolution of the ground state wave function of the upper surface of the diagonalized potential. The evolution on BO surface 2 is plotted.

\section{OPTIMAL CONTROL}

Locking is envisioned as a two-step process consisting of (1) transferring the initial ground state wave function to the position where it is to be locked and then (2) applying the constant amplitude locking field. To achieve the transfer a target wave function needs to be chosen. The parameters of Fig. 5 are such that the slope values correspond to the diatomic molecule CsI at the point where the BO surfaces have the same slope, but with opposite sign. The upper CsI BO state is repulsive, as seen from Table II. The locking point on the CsI BO surface is at $x_{0}=6.52 \mathrm{a} . \mathrm{u}$. Parameters for the CsI BO surfaces are given Table II.

The initial ground vibrational state for CsI was found by the method of Kosloff and Tal-Ezer [50] through integration of the time-dependent Schrödinger equation in imaginary time.

For the initial trial electric field we chose the chirped electric field from an earlier optimization of CsI [32]. The reason for this choice was that this chirped electric field results in a wave function that overlaps with the target wave function on both the lower as well as the upper surfaces and this choice ensures that a gradient method can start.

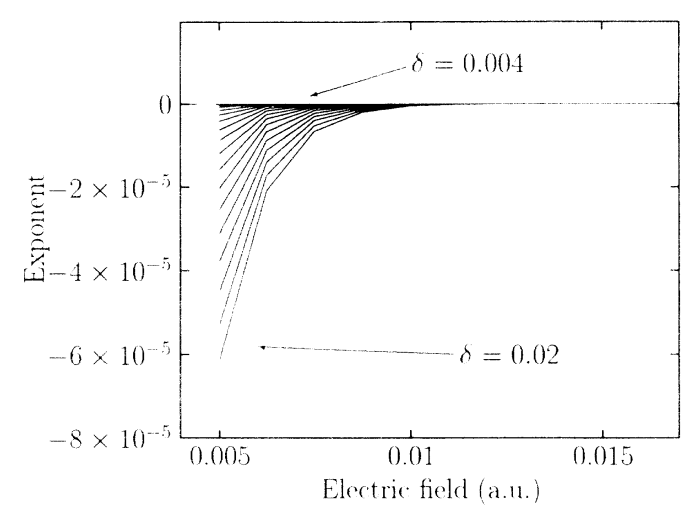

FIG. 6. Decay rates of locked wave functions vs electric field strengths. Parameters of the curves correspond to the slopes of the linear potentials in atomic units.

In the first iterations the Krotov method was used with the split operator (SPO) propagator [46,47]. The combination gave quick convergence; however, at later steps of the computation the method started to increase the electric field values for small penalties on the electric field energy and for the first few femtoseconds. Using a higher penalty value the effect became smaller. The conjugate gradient method (CGM) combined with a line search [31] is very slow compared to the Krotov method, and a mixed approach was tried by transferring the reasonable Krotov results to the CGM method for the case of low penalty and transferring the converged Krotov results to CGM for the case of high penalty. CGM improved the results for both cases, that is, the Krotov local maximum was smaller than the CGM one for this computation. For the final CGM runs, the weighted penalty function was included. The results on the electric field for the low penalty case is given in Fig. 7. The final time wave function for the low penalty case is displayed in Fig. 8. It is interesting to note that in the high penalty case almost the entire amplitude of the wave function is placed on one of the potentials and not on both as the target was. (The symmetry of the problem indicates that the actual potential surface where the wave function is placed to depends on the initial electric field.) The low penalty

TABLE II. Parameters of potentials and grid used in the simulation of CsI dynamics (as given in $[49])$.

\begin{tabular}{ll}
\hline \multicolumn{1}{c}{ Ground potential } & Excited potential \\
\hline$V_{g}=a \exp (-\beta r)-1 /\left(r-r_{1}\right)$ & $V_{u}=\exp \left[-d\left(r-r_{e}\right)\right]-C_{6} /\left(r-r_{2}\right)^{6}+V_{\infty}$ \\
$a=131.569$ a.u. & $d=2.57102 \mathrm{a} . \mathrm{u}$. \\
$\beta=1.37042 \mathrm{a} . \mathrm{u}$. & $r_{e}=4.20 \mathrm{a} . \mathrm{u}$. \\
$r_{1}=0.893631$ a.u. & $C_{6}=10.0 \mathrm{a} . \mathrm{u}$. \\
& $r_{2}=1.88972 \mathrm{a} . \mathrm{u}$. \\
& $V_{\infty}=-0.02939921$ a.u. \\
\hline Dipole moment & \\
$\quad \mu=0.3$ a.u. & \\
Masses & \\
$\quad m_{C s}=132.909 \mathrm{amu}$ & $m_{\mathrm{I}}=126.904 \mathrm{amu}$ \\
\hline Grid & $\Delta r=0.0165$ a.u. \\
$\quad N=256-512$ & $\Delta r$ \\
\hline \hline
\end{tabular}




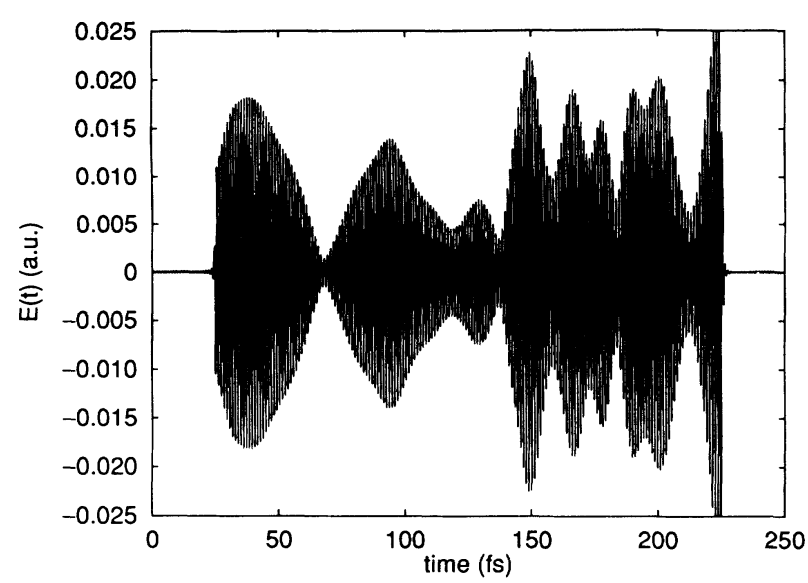

FIG. 7. Optimized electric field for low penalty $\left(\lambda_{e}=0.025\right)$.

solution, on the other hand, is powerful enough to place amplitudes to both BO surfaces as desired.

The resulting CsI wave packet of Fig. 8 was then locked at the same point with a sinusoidal field of $0.01 \mathrm{a}$.u. in strength. The probability of finding the system in the target state vs time is shown in Fig. 9. Close to $50 \%$ of the CsI stays in the target state (see the time dependence of the expectation value of the projector defined by the target state $\langle P\rangle$ in Fig. 9). In fact, almost $100 \%$ stays locked, but it oscillates around the target (see the time dependence of the expectation value of the projection into the region $6.3-7.0$ a.u. given by $P_{r}$ and the mean position values of the ground and upper state wave functions $\langle R\rangle_{g}$ and $\langle R\rangle_{u}$, respectively in Fig. 9). Figure 9 also shows that the populations on the upper and ground surfaces oscillate out of phase. The physical reason for this can be seen in the schematic of Fig. 10, which illustrates the nature of locking to be a recycling of the wave packet by the field between the upper and ground potentials. The small amount of leakage found corresponds to those portions of the wave packet that escape the recycling process and exit to the right on the upper curve and to the left on the ground curve.

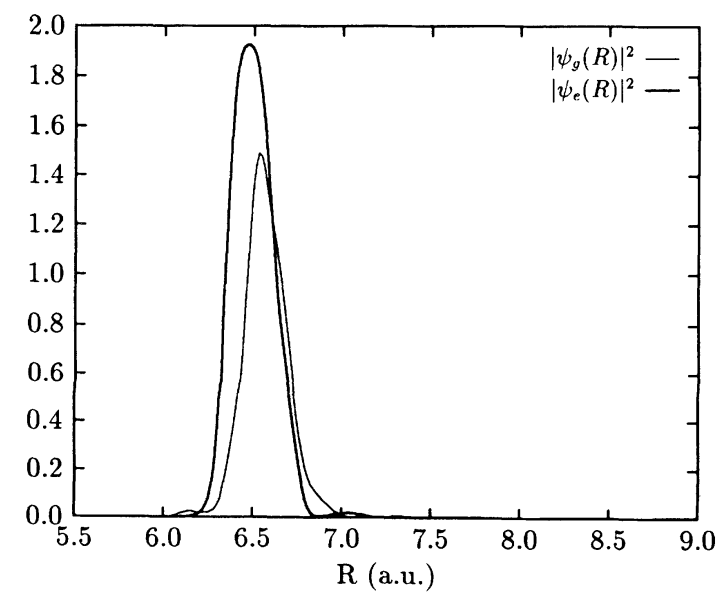

FIG. 8. Final time wave function around target position for the low penalty case.
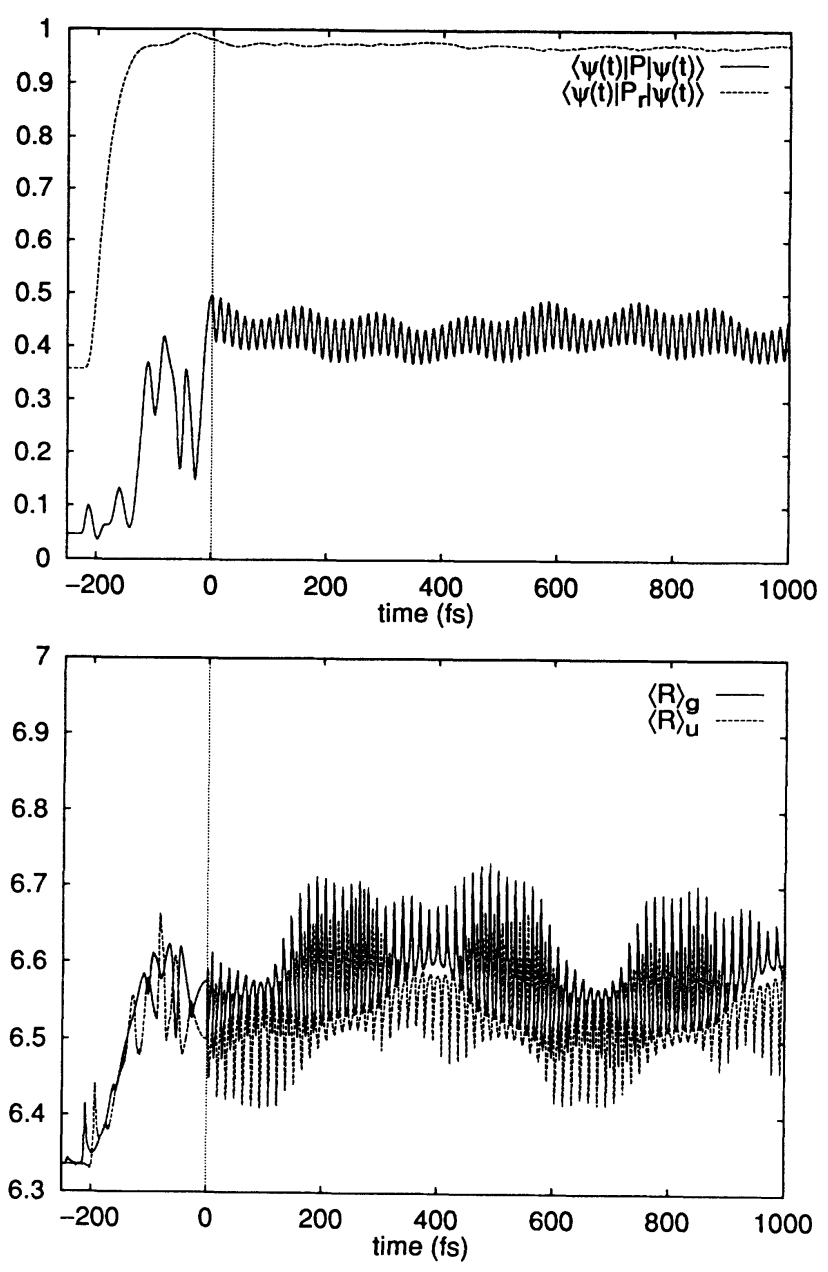

FIG. 9. Results of locking. The continuous curve of the upper figure shows the time dependence of the probability of finding the CsI system in the target state. The dashed curve of the upper figure plots the time dependence of the expectation value of finding the wave function in the region between 6.3 and 7.0 a.u. Curves of the lower figure show the position mean values of the ground $\left(\langle R\rangle_{g}\right)$ and upper potential wave functions $\left(\langle R\rangle_{u}\right)$, respectively. Time zero corresponds to the end of the optimal control procedure to create the target wave function and to the start of sinusoidal locking field.

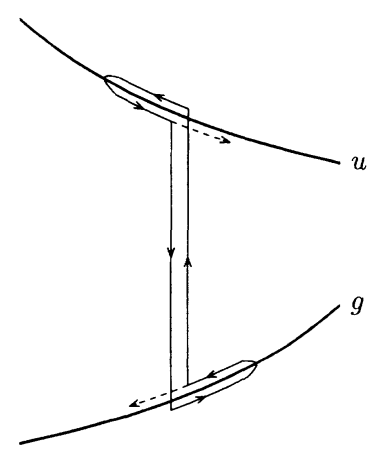

FIG. 10. Schematic of locking corresponding to wave-packet recycling according to the indicated path between the two surfaces $u$ and $g$. The dashed arrows correspond to the small portions of the wave packet that escape the locking process. 


\section{CONCLUSION}

The objective of this work is to extend optimal control theory to prepare and hold molecules in quasistationary nonequilibrium states, with the ultimate goal of subsequently studying the properties of such molecular configurations. A variety of possible applications of locking can be envisioned including to the scattering and spectroscopic properties of such molecules. In addition, the ability to locally lock molecular configurations at a sequence of structures combined with laboratory feedback optimal control $[23,32,33,44]$ may open up a new approach to potential surface determination. We have shown that one can prepare a given state localized at a given bond length with the help of the optimal control approach and that the resulting state can be successfully locked with the help of a constant amplitude sinusoidal electric field. Locking may be made efficient and long lasting with reasonable strength electric fields. It is, however, clear that control theory is not being fully utilized in this example. Control is used here only to prepare the wave packet that can be locked efficiently with a sinusoidal electric field.
Control theory, however, could be used to optimize locking over the full time period. In this respect the work may be considered as providing the initial guess for a full optimal control study. On the other hand, we would like to stress that the approach is viable from the experimental point of view when the process is to be optimized in the laboratory and the number of optimizing parameters should be kept low: The approach restricts the long time control to the optimization of a few parameters, e.g., the amplitude, the frequency, and possibly the chirp of the electric field [32].

\section{ACKNOWLEDGMENTS}

Part of this work was supported by The Danish Natural Research Science Council, by OTKA Grant of the Hungarian Academy of Science (OTKA 1890/1991), and by the U.S.-Hungarian Joint Fund (JF 168). H.R. acknowledges support from the Army Research Office. B.A. wishes to thank Danmarks Tekniske Højskoles Fond for Teknisk Kemi for travel support to Hungary.
[1] D.J. Tannor and S.A. Rice, J. Chem. Phys. 83, 5013 (1985).

[2] D.J. Tannor, R. Kosloff, and S.A. Rice, J. Chem. Phys. 85, 5805 (1986).

[3] M. Shapiro and P. Brumer, J. Chem. Phys. 84, 4103 (1986).

[4] P. Brumer and M. Shapiro, Faraday Discuss. Chem. Soc. 82, 177 (1986).

[5] P. Brumer and M. Shapiro, Chem. Phys. Lett. 126, 541 (1986)

[6] A.P. Peirce, M.A. Dahleh, and H. Rabitz, Phys. Rev. A37, 4950 (1988).

[7] S. Shi, A. Woody, and H. Rabitz, J. Chem. Phys. 88, 6870 (1988).

[8] S. Shi and H. Rabitz, Chem. Phys. 139, 185 (1989).

[9] R. Kosloff et al., Chem. Phys. 139, 201 (1989).

[10] M.A. Dahleh, A.P. Peirce, and H. Rabitz, Phys. Rev. A 42, 1065 (1990).

[11] S. Shi and H. Rabitz, J. Chem. Phys. 92, 364 (1990).

[12] S. Shi and H. Rabitz, J. Chem. Phys. 92, 2927 (1990).

[13] S.H. Tersigni, P. Gaspard, and S.A. Rice, J. Chem. Phys. 93, 1670 (1990).

[14] K. Yao, S. Shi, and H. Rabitz, Chem. Phys. 150, 373 (1990).

[15] W. Jakubetz, B. Just, J. Manz, and H.-J. Schreier, J. Phys. Chem. 94, 2294 (1990).

[16] W. Jakubetz, J. Manz, and H.-J. Schreier, Chem. Phys. Lett. 165, 100 (1990).

[17] G.K. Paramonov, Chem. Phys. Lett. 169, 573 (1990).

[18] B. Amstrup, R. Carlson, A. Matro, and S.A. Rice, J. Phys. Chem. 95, 8019 (1991).

[19] C.D. Schwieters and H. Rabitz, Phys. Rev. A 44, 5224 (1991).

[20] C.K. Chan, P. Brumer, and M. Shapiro, J. Chem. Phys. 94, 2688 (1991).
[21] J.G.B. Beumee and H. Rabitz, J. Chem. Phys. 97, 1353 (1992).

[22] P. Gross, D. Neuhauser, and H. Rabitz, J. Chem. Phys. 96, 2834 (1992).

[23] R.S. Judson and H. Rabitz, Phys. Rev. Lett. 68, 1500 (1992).

[24] R. Kosloff, A.D. Hammerich, and D. Tannor, Phys. Rev. Lett. 69, 2172 (1992).

[25] S. Shi and H. Rabitz, J. Chem. Phys. 97, 276 (1992).

[26] D.J. Tannor, V. Kazakov, and V. Orlov, in Time Dependent Quantum Molecular Dynamics, edited by J. Broeckhove and L. Lathouwers (Plenum Press, New York, 1992), pp. 347-360.

[27] B. Just, J. Manz, and I. Trisca, Chem. Phys. Lett. 193, 423 (1992).

[28] B. Just, J. Manz, and G.K. Paramonov, Chem. Phys. Lett. 193, 429 (1992).

[29] J.E. Combariza, S. Görtler, B. Just, and J. Manz, Chem. Phys. Lett. 195, 393 (1992).

[30] B. Amstrup and N.E. Henriksen, J. Chem. Phys. 97, 8285 (1992).

[31] B. Amstrup, A. Lörincz, and S.A. Rice, J. Phys. Chem. 97, 6175 (1993).

[32] B. Amstrup et al., Phys. Rev. A 48, 3830 (1993).

[33] P. Gross, D. Neuhauser, and H. Rabitz, J. Chem. Phys. 98, 4557 (1993).

[34] P. Gross, D. Neuhauser, and H. Rabitz, J. Chem. Phys. 98, 9650 (1993).

[35] P. Gross et al., Phys. Rev. A 47, 4593 (1993).

[36] M. Shapiro and P. Brumer, Chem. Phys. Lett. 208, 193 (1993).

[37] L. Shen, S. Shi, and H. Rabitz, J. Phys. Chem. 97, 8874 (1993).

[38] L. Shen et al., J. Chem. Phys. 98, 7792 (1993).

[39] J. Somlói, V.A. Kazakov, and D.J. Tannor, Chem. Phys. 
172, 85 (1993).

[40] C.D. Schwieters, K. Yao, and H. Rabitz, J. Phys. Chem. 97, 8864 (1993).

[41] T. Szakács, J. Somlói, and A. Lörincz, Chem. Phys. 172, 1 (1993).

[42] T. Szakács, T. Lucza, and A. Lörincz, Surf. Sci. 296, 251 (1993).

[43] Y. Yan et al., J. Phys. Chem. 97, 2320 (1993).

[44] W.S. Warren, H. Rabitz, and M. Dahleh, Science 259, 1581 (1993).

[45] D.G. Luenberger, Linear and Nonlinear Programming, 2nd ed. (Addison-Wesley, New York, 1984).

[46] C. Leforestier et al., J. Comput. Phys. 94, 59 (1991).

[47] M.D. Feit, Jr., J.A. Fleck, and A. Steiger, J. Comput. Phys. 47, 412 (1982).

[48] R. Kosloff and D. Kosloff, J. Comput. Phys. 63, 363 (1986).

[49] S. Ruhman and R. Kosloff, J. Opt. Soc. Am. B 7, 1748 (1990).

[50] R. Kosloff and H. Tal-Ezer, Chem. Phys. Lett. 127, 223 (1986). 


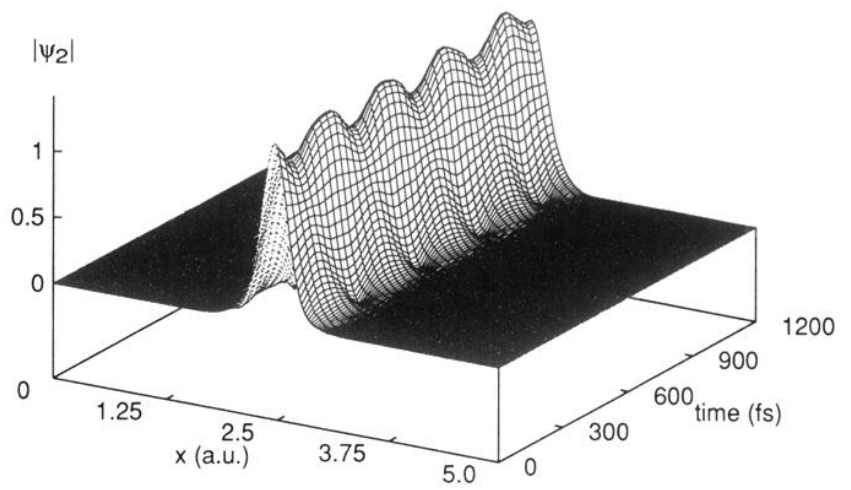

FIG. 1. Evolution of the "cleaned" wave function on BO surface 2. Evolution on BO surface 1 is similar, but opposite in phase. 


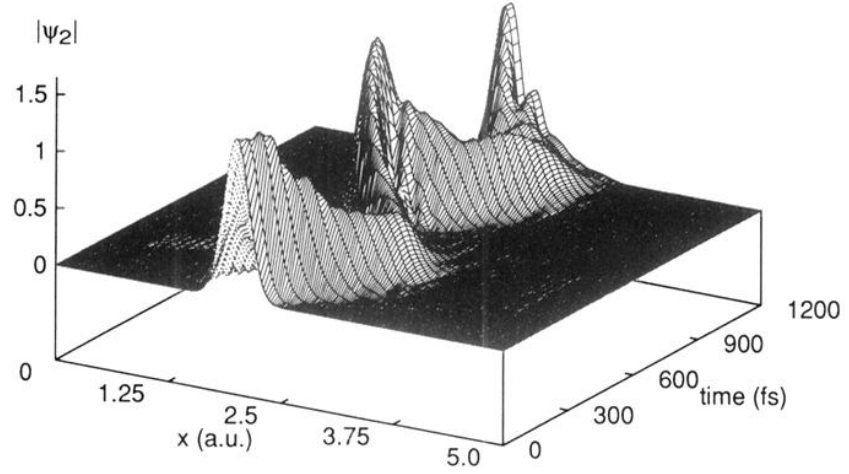

FIG. 3. Evolution of displaced wave function on BO surface 2 . 


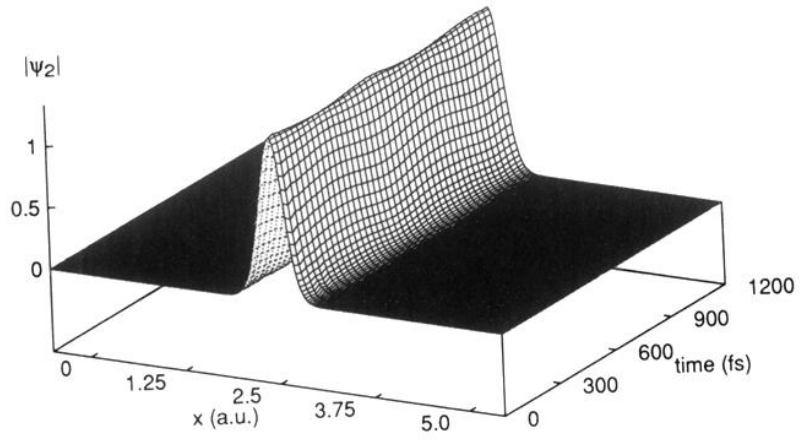

FIG. 5. Evolution of the ground state wave function of the upper surface of the diagonalized potential. The evolution on BO surface 2 is plotted. 


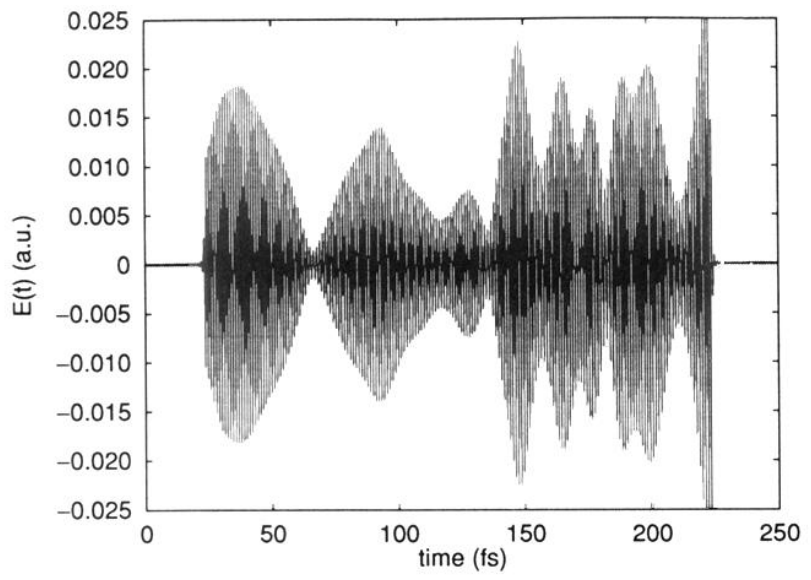

FIG. 7. Optimized electric field for low penalty $\left(\lambda_{e}=0.025\right)$. 